$\overline{A E E T}$

ASOCIACIÓN ESPAÑOLA

DE ECOLOGÍA TERRESTRE

\title{
La composición y propiedades mecánicas de cuernas y huesos de ciervo como fuente de información para gestionar ecosistemas
}

\author{
T. Landete-Castillejos ${ }^{1,2,3}$, A. Garcia ${ }^{1,2,3}$, F. Ceacero ${ }^{4}$, L. Gallego $0^{1,2,3}$
}

(1) Grupo de Ciencia Animal Aplicada a la Gestión de la Fauna, Instituto de Investigación en Recursos Cinegéticos, IREC (CSIC-UCLM-JCCM). Campus UCLM Albacete 02071, Albacete, España

(2) Escuela Superior de Ingenieros Agrónomos de la Universidad de Castilla-La Mancha, Campus Albacete, 02071 Albacete, España.

(3) Sección de Recursos Cinegéticos, Instituto de Desarrollo Regional, Campus Albacete de la UCLM, 02071 Albacete, España.

(4) Department of Animal Science and Food Processing. Faculty of Tropical AgriSciences. Czech University of Life Sciences. Prague, República Checa.

* Autor de correspondencia: T. Landete-Castillejos [Tomas.Landete@uclm.es]

> Recibido el 31 de enero de 2013, aceptado el 23 de julio de 2013.

Landete-Castillejos, T., Garcia, A., Ceacero, F., Gallego, L. (2013). La composición y propiedades mecánicas de cuernas y huesos de ciervo como fuente de información para gestionar ecosistemas. Ecosistemas 22(2):68-75. Doi.: 10.7818/ECOS.2013.22-2.11

Este estudio hace una revisión de una línea de investigación que muestra cómo, en el ciervo, la composición mineral, propiedades mecánicas del material óseo de la cuerna, estructura e incluso histología de las cuernas están influidos por un juego de factores interrelacionados tales como la nutrición, tipo de gestión, calidad del hábitat, y densidad de población. Así, evaluar estas características se convierte en una herramienta diagnóstica que aporta información sobre las deficiencias minerales, calidad del hábitat y otras que podría ser usada para gestionar el ecosistema. Se muestran resultados de estudios en huesos internos, y las implicaciones para la gestión de especies que no tienen cuernas.

Palabras clave: Cervus elaphus, composición mineral, gestión cinegética, nutrición, propiedades mecánicas intrínsecas

Landete-Castillejos, T., Garcia, A., Ceacero, F., Gallego, L. (2013). The composition and mechanical properties of deer antlers and bones as an information source to manage ecosystems. Ecosistemas 22(2):68-75. Doi.: 10.7818/ECOS.2013.22-2.11

This study reviews a line of research showing how deer antler mineral composition, mechanical properties of the bone material, structure, and even histology are influenced by a range of interrelated factors such as nutrition, type of management, habitat quality and population density. Thus, assessing these characteristics becomes a diagnostic tool providing information regarding mineral deficiencies, habitat quality, and others that may be used for managing the ecosystem. Results are also shown for studies on internal bones, and the implications for management of species that do not grow antlers.

Keywords: Cervus elaphus, game management, intrinsic mechanical properties, mineral composition, nutrition

\section{Gestión cinegética y gestión para la conservación}

La historia de la gestión cinegética y la gestión de ecosistemas para su conservación está plagada de ejemplos de buena y mala gestión. Históricamente, los grandes parques nacionales de Europa, desde Doñana en España a Bialowieza en Polonia, han surgido del hecho de que eran reservas de caza reales o de la nobleza. Anteriormente, incluso, los papiros de Egipto muestran escenas de caza que el faraón sólo podía asegurarse prohibiéndola a quien él no autorizase (curiosamente, muchas tumbas muestran escenas de caza, lo que indica lo importante que era esta actividad para el faraón). Las reservas de caza reales, precursoras de muchos parques nacionales, se crean por que la propia caza por el ser humano puede reducir las poblaciones animales en ocasiones hasta su práctica extinción. Sin acudir a la historia, la caza organizada por varios socios en un coto suele tener como primera regla que los socios sean fiables y acuerden cupos de caza, ya que puede suceder en cotos con muchos cazadores que los menos fiables se salten esta norma. Esta dualidad se ha ido dando a lo largo de la historia (por que junto al hecho de preservar las áreas de caza se ha tratado de extinguir a los depredadores de esa caza -y con éxito en algún caso-) hasta el día de hoy. Actualmente se critica la gestión cinegética como algo poco natural frente a la gestión que se lleva a cabo en parques naturales o nacionales. Esto en parte es cierto ya que por un lado, la gestión cinegética entendida como una actividad económica sin límites éticos produce, por un lado, la liberación en el medio natural de perdiz roja (Alectoris rufa) criada en granja en España (o faisanes en Alemania), y por otro la creación de vallados pequeños o cercones cuyo objetivo es el de proporcionar una caza fácil y de alta rentabilidad, con animales procedentes de granjas. Sin embargo, la gestión cinegética bien entendida puede, por ejemplo, convertir la seca llanura manchega en una de las zonas húmedas más importantes de España (Dehesa de Monreal, en Toledo, un conjunto de 14 lagunas extendiéndose sobre $200 \mathrm{Ha}$ con un censo de más de 10000 aves al mes), o algunos cotos de caza mayor en auténticos parques naturales donde crían con más tranquilidad las especies más amenazadas de rapaces o incluso el lince. En ambos casos, se trata de conservación a 
coste cero para el estado. Si logramos que una gestión cinegética con un enfoque de conservación del medio sea más rentable que dedicar esas tierras a la agricultura, entonces la conservación del medio aumentará por las mismas fuerzas que hacen crecer la economía de los países. En ese proceso puede ayudar la ciencia tal como se indica en la presente revisión. El papel de los legisladores será crear el marco en el que sólo sea posible una gestión cinegética compatible con la conservación, o bien hacer que este enfoque sea más rentable que el de la caza intensiva y artificial.

\section{El uso de las cuernas como fuente de datos en gestión cinegética}

La gestión cinegética en caza mayor tiene por lo general un objetivo claro: mejorar los trofeos. Esto ha llevado tradicionalmente a seguir dos caminos. Uno inmediato y poco ético es traer ciervos, jabalíes, etc. de gran tamaño pero de origen alóctono. El otro camino es mejorar el hábitat (aumentando el alimento por cabeza a través de la reducción de la densidad de población, o añadiendo suplementos alimenticios o minerales). Tradicionalmente, la gestión cinegética del segundo tipo se ha basado en los conocimientos basados en la experiencia de quien ha estado toda la vida en el campo (los guardas, los encargados de las fincas, etc.). Sin embargo, quienes se dedican a gestionar fincas, e incluso los que sólo son cazadores, hace ya años que se han dado cuenta de que la gestión cinegética debe tener una base científica. Esto no sólo es más eficiente porque se usan los hallazgos más nuevos, sino que en muchos casos es más barato ya que no hace falta aportar los interminables camiones de pienso y correctores que se usaban hace años en algunos cotos (y que convertían a la caza mayor en un nuevo tipo de ganado).

La ciencia necesita datos empíricos para llegar a conclusiones válidas, y la obtención de datos en el campo se ha hecho a partir de muestras tomadas en monterías, en cacerías selectivas, en algunos casos hechas a la medida de las necesidades de los científicos, o a partir de datos tomados por los propios cazadores. Hace unos años nuestro grupo de investigación pensó en usar como fuente de datos la cuerna de los ciervos (cérvidos en general, ya que podría usarse también la de corzos, gamos y otras especies). Como ventajas, las cuernas: $1^{\circ}$ ) son estables y pueden recogerse en el campo a lo largo del invierno y primavera sin que la climatología las afecte seriamente (sólo se deterioran y se vuelven grises cuando llevan más de un año en el campo); $2^{\circ}$ ) como todos los animales pierden las cuernas cada año, se puede tener en principio datos de toda la población sin necesidad de matarlos o capturarlos; esto evita sesgos en la muestra ya que ningún gestor abatiría a un buen trofeo para uso científico; $3^{\circ}$ ) las cuernas se venden al peso para cuchillería y otros usos, por lo que los guardas buscan con gran interés los desmogues (o sea, para hacer un estudio se pueden medir todas las cuernas de la población, a partir de ahí seleccionar una muestra representativa, y dejar que el resto se venda); $4^{\circ}$ ) las cuernas guardan sus propiedades y su información durante mucho tiempo, y muchos gestores guardan cuernas de cada año, con lo que es posible hacer estudios históricos muy interesantes para los científicos y para los propios dueños del coto; $5^{\circ}$ ) la información aportada es útil para los gestores ("tu ciervo tiene esta y esta otra deficiencia mineral"), pero ni le crea un problema sanitario ni de homologación de trofeos debido a una potencial contaminación genética, de modo que todo el mundo quiere colaborar (y de hecho, gestores de España y otros países europeos están dispuestos a pagar por hacer estos estudios).

\section{Las base biológica que convierte a las cuernas en una gran fuente de información}

Las cuernas de venado, además de un trofeo deseado, son una de las estructuras más increíbles de los animales. Son el único hueso que crece fuera del cuerpo, y esto significa que es de fácil acceso para estudiar la biología del hueso. Una característica que tarde o temprano las convertiría en útiles para estudios médicos. No sólo eso, crecen y caen cada año. Esto las hace muy costosas. En 1931 Sir Julian Huxley determinó que la cuerna constituye del 1 al $5 \%$ del peso corporal del ciervo (Huxley 1931). Nuestro grupo demostró además que la cuerna constituye el $28 \%$ del peso del esqueleto en ciervos adultos (Gomez et al. 2012). Es decir, que el $28 \%$ del peso del esqueleto crece y cae cada año. Es un enorme esfuerzo.

La velocidad de crecimiento del tejido precursor de la cuerna es la mayor de cualquier tejido animal (salvo el cáncer), variando de 0.5 a $4 \mathrm{~cm} / \mathrm{d}$ (ver Goss 1983); así, en ciervos ibéricos adultos, el promedio es de $0.51 \mathrm{~cm} / \mathrm{d}$ a partir de los datos en Gaspar-Lopez et al. (2010), y 0.68 cm/d según Gómez et al. (2013). Esta velocidad es tan rápida que la alimentación no logra aportar la cantidad de minerales necesarios para la formación de la cuerna, de modo que un ciervo tiene que transferir un $28 \%$ de su esqueleto óseo hacia las cuernas (ver Muir et al. 1987). Esto produce un proceso similar a la osteoporosis en los seres humanos, con la única diferencia de que es reversible: los ciervos sufren este proceso de osteoporosis durante el crecimiento de la cuerna (primavera y verano de cada año), y se recuperan más tarde a lo largo del otoño e invierno (Baxter et al. 1999). Este hecho inspiró la idea de que los estudios sobre cuerna podrían ayudar a entender esta enfermedad. Posteriormente, esta idea se plasmó en una hipótesis sobre el origen de esta enfermedad basada tanto en hallazgos sobre cuerna como en datos clínicos (Landete-Castillejos et al. 2012b). Las cuernas son por lo tanto estructuras costosas, pero los beneficios sobrepasan el esfuerzo de hacerlas crecer. Se ha demostrado que el tamaño de la cuerna en ciervos está relacionado con la dominancia, lo que implica mayor acceso a los recursos, tales como el alimento o la reproducción (Topinski 1974; Clutton-Brock et al. 1979, 1982, 1988). Como todos los ciervos hacen el máximo esfuerzo para producir la cuerna más grande, el esfuerzo máximo de un macho sano con buena condición corporal no puede ser comparable al esfuerzo máximo de un animal de calidad media o uno que esté enfermo, por la sencilla razón de que el primero puede conseguir la mejor comida o posee el mejor conjunto de genes. Por lo tanto, en nuestro grupo de investigación pensamos que las cuernas deben indicar el esfuerzo fisiológico, por lo que animales más capaces de realizar dicho esfuerzo deben tener parte o todas las características de la cuerna distintas de otros más débiles, con peor nutrición o enfermos; en otras palabras, pensamos que podríamos utilizar las cuernas para conocer el estado o condición de los animales. Con esta premisa evaluamos las características de las cuernas: examinando desde la composición mineral a la calidad mecánica del material óseo, desde la histología a la mecánica estructural (la resistencia no se debe exclusivamente al material, sino también al espesor de, por ejemplo, la pared de la cuerna).

\section{La composición de las cuernas y otros huesos re- fleja la dieta y la calidad del hábitat}

Los pasos que dimos para usar la cuerna como una herramienta diagnóstica fueron los siguientes: $1^{\circ}$ ) evaluar si hay diferencias en la composición y otras características de la cuerna entre distintos animales y regiones de la cuerna (sólo si había variación podía buscarse una relación con la gestión); $2^{\circ}$ ) Examinar diferencias producidas en las cuernas por los dos tipos de gestión y nutrición más alejados que teníamos a nuestra disposición (nutrición de alta calidad en la granja experimental de la UCLM frente a nutrición en un hábitat subóptimo para ciervos); $3^{\circ}$ ) evaluar si había cambios en un mismo coto entre dos años muy diferentes en climatología (es decir, un año bueno frente a uno malo).

La primera pregunta la contestamos realizando estudios de composición en la cuerna más sencilla (la vara de varetos o cuerna de animales de un año) comparando la base y la punta de la cuerna y diferencias entre distintos animales que estuvieran relacionadas con variables que afectaban a la calidad del individuo, como su peso corporal y crecimiento durante la lactación y post-lactación hasta el periodo de crecimiento de la cuerna (Landete-Castillejos 
et al. 2007a). Los resultados mostraron que había diferencias entre la base y la punta que posteriormente se reflejaban en otros estudios (Landete-Castillejos et al. 2007b, 2010a). Estas diferencias parecen estar relacionadas con el esfuerzo fisiológico de hacer crecer las cuernas: hay más hierro, potasio y zinc en la punta que en la base. En otros estudios, a menudo hay mayor concentración de estos minerales en animales con peor alimentación y no sólo en cuernas, sino en huesos internos (Olguin et al. 2013). La comparación entre distintos individuos indicó que podíamos explicar las variables tradicionalmente relacionadas con la calidad de la cuerna, el peso y la longitud, que a su vez están relacionadas con el tamaño corporal del animal, a partir de unos pocos minerales, los cuales explicaban en torno al $75 \%$ de la variabilidad (Landete-Castillejos et al. 2007a). En el caso del peso, sólo el potasio explicaba el $40 \%$ de la variación en peso; esto es, había una composición propia de cuernas de buena o mala calidad. Por otra parte, el crecimiento durante la lactación era más importante para explicar la composición de los minerales mayoritarios de la cuerna, que el crecimiento entre la lactación y el crecimiento de la cuerna (Landete-Castillejos et al. 2007a).

En relación a la segunda pregunta planteada, una vez establecida la variación en composición mineral y la relación con otras variables, el siguiente estudio demostró que la gestión podía influir en las características de la cuerna, y no sólo en la composición sino en las propiedades mecánicas e histología (Landete-Castillejos et al. 2007b). La situación de gestión/nutrición para el ciervo más extremas se establecieron en las siguientes localizaciones: a) condición óptima, granja de ciervos de la UCLM, en Albacete (España), en donde la alimentación se caracteriza por el aporte de un porcentaje de proteína del $16 \%$, los animales se desparasitan periódicamente, estos presentan poco gasto energético al encontrarse estabulados, y no hay necesidad de competir por la comida, la cual se aporta ad libitum; b) un coto de la administración regional situado en Alpera (Albacete, España), en donde se ha determinado que la proteína disponible para los ciervos es del $7 \%$, existe competencia con otras especies animales (otros ungulados y conejos), no hay tratamiento veterinario, y el hábitat es considerado subóptimo para el ciervo al carecer de ciertas especies vegetales consideradas esenciales en la dieta del ciervo, tales como las jaras Cistus sp. (Gebert y Verheyden-Tixier 2001). Los resultados mostraron que la cuerna sirve como índice para evaluar la gestión y nutrición en todas las propiedades analizadas. La composición media de la cuerna reflejaba la nutrición (Estevez et al. 2009; Tabla 1), de modo que aquellos minerales que mostraban una composición significativamente diferente entre cuernas de distinto origen (sodio, potasio, y manganeso) también mostraban en ese orden una mayor diferencia entre la concentración en la dieta de alta calidad (de la granja) y la más pobre del coto $(6.3,3.1$ y 2.2 , respectíva-

Tabla 1. Composición mineral de cuernas de ciervos de granja (UCLM) alimentados con una dieta rica en proteína (16\%) y minerales, y ciervos silvestres en un coto con un habitat subóptimo (proteína 7 \%). La columna de la derecha muestra el ratio entre la concentración del nutriente en la dieta de ciervos de granja frente a la de ciervos silvestres.

\begin{tabular}{lcccc}
\hline & $\begin{array}{c}\text { Ciervos } \\
\text { silvestres }\end{array}$ & $\begin{array}{c}\text { Ciervos de } \\
\text { granja }\end{array}$ & P & $\begin{array}{c}\text { Ratio concentración } \\
\text { en dieta de ciervos de } \\
\text { granja / silvestres }\end{array}$ \\
\hline Proteína (\%) & $36.6 \pm 0.2$ & $35.3 \pm 0.3$ & 0.007 & 1.8 \\
$\mathrm{Ca}(\%)$ & $34.5 \pm 0.2$ & $35.0 \pm 0.3$ & - & 1.5 \\
$\mathrm{Na}(\%)$ & $0.92 \pm 0.02$ & $1.15 \pm 0.02$ & 0.001 & 6.3 \\
$\mathrm{Mg}(\%)$ & $0.81 \pm 0.03$ & $0.99 \pm 0.02$ & 0.001 & 3.1 \\
$\mathrm{~K}(\mathrm{mg} / \mathrm{kg})$ & $661 \pm 30$ & $864 \pm 46$ & 0.001 & 2.2 \\
$\mathrm{Fe}(\mathrm{mg} / \mathrm{kg})$ & $68 \pm 7$ & $50 \pm 5$ & - & 1.6 \\
$\mathrm{Zn}(\mathrm{mg} / \mathrm{kg})$ & $98 \pm 4$ & $95 \pm 4$ & - & 1.2 \\
\hline
\end{tabular}

mente para el sodio, potasio, y manganeso). Una deficiencia mineral no sólo se mostraba en las medias, sino también en la evolución de la concentración de minerales (Landete-Castillejos et al. 2007b). Así, por ejemplo, en las cuernas de ciervos bien alimentados, el sodio mostraba una concentración estable de base o roseta a punta de una cuerna (Fig. 1), mientras que los que tenían una alimentación peor en campo mostraban una composición de sodio que descendía conforme avanzaba la formación de la cuerna (o sea, de la base a la punta). El manganeso mostraba un efecto similar, aunque en otros minerales la evolución era más compleja o de sentido contrario (como el potasio, indicando que su concentración aumenta conforme el animal hace un esfuerzo fisiológico mayor y va agotando la reserva movilizable de minerales de su esqueleto).

Este efecto era similar en todas las propiedades mecánicas analizadas (Landete-Castillejos et al. 2007c). Antes de mostrar dicho efecto, conviene explicar brevemente un par de conceptos básicos en mecánica. Una de las características de las cuernas que las hacen especialmente interesantes para los expertos en mecánica ósea es que está considerado el hueso más resistente (Currey et al. 2009). Las cuernas rara vez se rompen y en los pocos casos en que lo hacen (por lo general menos del $10 \%$ ) lo hacen en las puntas (Landete-Castillejos et al. 2010a). La resistencia a la rotura de una cuerna o cualquier estructura puede dividirse en dos factores (Currey 2002): a) Resistencia arquitectónica o derivada de tener las paredes más gruesas, mayor diámetro, etc. (lo que puede explicar el $55 \%$ del rendimiento mecánico de un hueso, Davison et al. 2006); esta variable nos puede explicar si hay problemas de gestión, dado que en nuestro ejemplo, las cuernas de hábitat subóptimo tenían un espesor cortical mucho menor que las de granja (Estevez et al. 2008, y Fig. 2). b) Calidad mecánica del material (no es igual de resistente un tubo de acero que uno de plástico, aunque el diámetro y pared sean iguales); en donde distinguimos las propiedades mecánicas denominadas intrínsecas, entre otras, la rigidez o módulo de Young (fuerza necesaria para producir una deformación del $1 \%$ ), la fuerza de rotura (fuerza necesaria para romper el material), y el trabajo de fractura (Currey 2002). Nuestro estudio mostró dos características muy similares a lo que sucedía con muchos minerales (Landete-Castillejos et al. 2007c): $1^{\circ}$ ) Las propiedades mecánicas del material decrecían de la base a la punta en ambas poblaciones, indicando de nuevo un esfuerzo fisiológico; y $2^{\circ}$ ) el material de las cuernas con una alimentación óptima tenía una calidad mayor a cualquier nivel que el de las cuernas de ciervos del hábitat subóptimo.

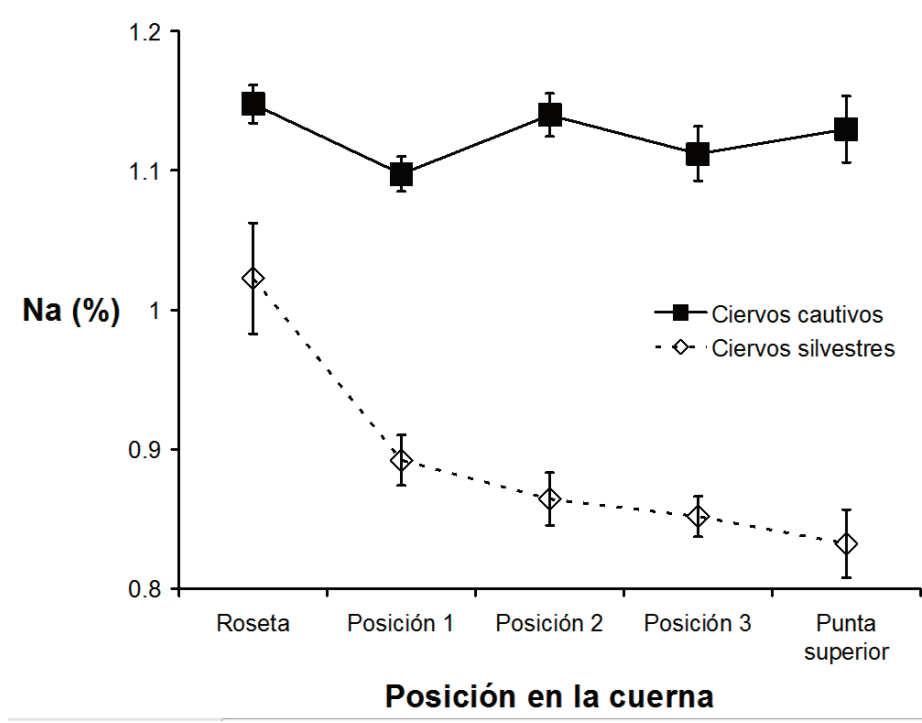

Figura 1. Contenido en sodio ( $\mathrm{Na}$ ) a lo largo de la vara principal de cuernas de ciervo adulto desde la base (roseta) a la punta (de izquierda a derecha). La línea sólida corresponde a ciervos de la granja de la UCLM con alimentación de alta calidad (16 \% proteína). La línea punteada corresponde a ciervos silvestres en un hábitat subóptimo (6-7\% de proteína). 


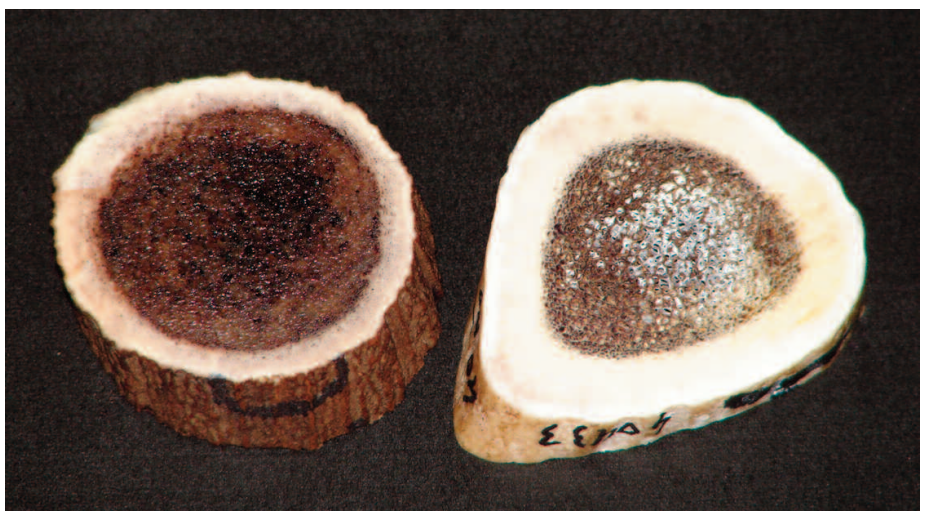

Figura 2. Cuernas de ciervos de granja (UCLM) mantenidos con una alimentación de alta calidad (16\% de proteína) mostrando un notable espesor de la pared exterior (derecha) frente a las cuernas de ciervos silvestres en un hábitat subóptimo con una alimentación más pobre (7 \% proteína) (izquierda).

Asimismo se ha demostrado que la gestión de la población o la calidad nutritiva que reciben los ciervos afecta al aspecto histológico del tejido óseo de las cuernas (Landete-Castillejos et al. 2012a): la porosidad aumentaba de nuevo de la base a la punta, y era cinco veces mayor en los ciervos con una peor alimentación que en los de la granja (Fig. 3). El teñido del tejido para determinar la distribución y cantidad de zinc o potasio era consistente con los resultados de la porosidad. El zinc es un metal que se encuentra en buena medida unido a la fosfatasa alcalina, una enzima responsable de la mineralización. Por ello, era más abundante en osteonas (las varillas que forman el tejido óseo) mientras estaban mineralizando y desaparecía al estar totalmente mineralizadas. Este marcaje mostraba una mineralización completa en la base y partes medias de las cuernas de ciervos de granja, mientras que era más abundante en toda la cuerna de ciervos con peor alimentación y aumentaba de la base a la punta (Fig. 4). Landete-Castillejos et al. (2012a) también demostraron que el zinc, al igual que el potasio (ver más adelante), explicaba mejor la variación en propiedades mecánicas, en cierta forma porque se anticipaba a la aparición de la porosidad. El caso del potasio es un buen ejemplo de cómo un enfoque multidisciplinar, en donde participan profesionales de varias disciplinas, puede ayudar a producir hallazgos inesperados. Así, en un estudio en donde colaboraron ecólogos y expertos en anatomía patológica (Landete-Castillejos et al. 2012a), se demostró que el potasio se encuentra en el andamiaje sobre el que crecen las osteonas, de modo que abunda más cuanto menos desarrolladas están éstas; y al madurar el tejido dicho andamiaje es sustituido parcialmente (Fig. 4). Era la primera vez que se demostraba un papel del potasio en histología del hueso. Futuros estudios probablemente indiquen por qué el hierro y otros minerales parecen estar indicando también un cierto estrés fisiológico.

\section{Efectos de la calidad del hábitat en la cuerna}

Los huesos internos han evolucionado para ser rígidos y soportar nuestro peso corporal o transmitir casi toda la energía de los músculos en movimiento en lugar de absorber la energía de deformación. Por el contrario, las cuernas están diseñadas para atacar y empujar contra un oponente, sin romperse. El resultado es un hueso casi irrompible y prácticamente tan rígido como los huesos internos. De hecho Currey et al. (2009) demostraron que el material de cuerna en su estado fisiológico (seca) es 7 veces más difícil de romper que el material de un fémur (que está húmedo en estado fisiológico). Este dato ayuda a entender lo sorprendente del hecho acaecido en el año 2005, cuando se constató un elevado número de roturas de cuernas en varias fincas cinegéticas españolas (algo que se ha producido de nuevo en el invierno de 2012-2013).

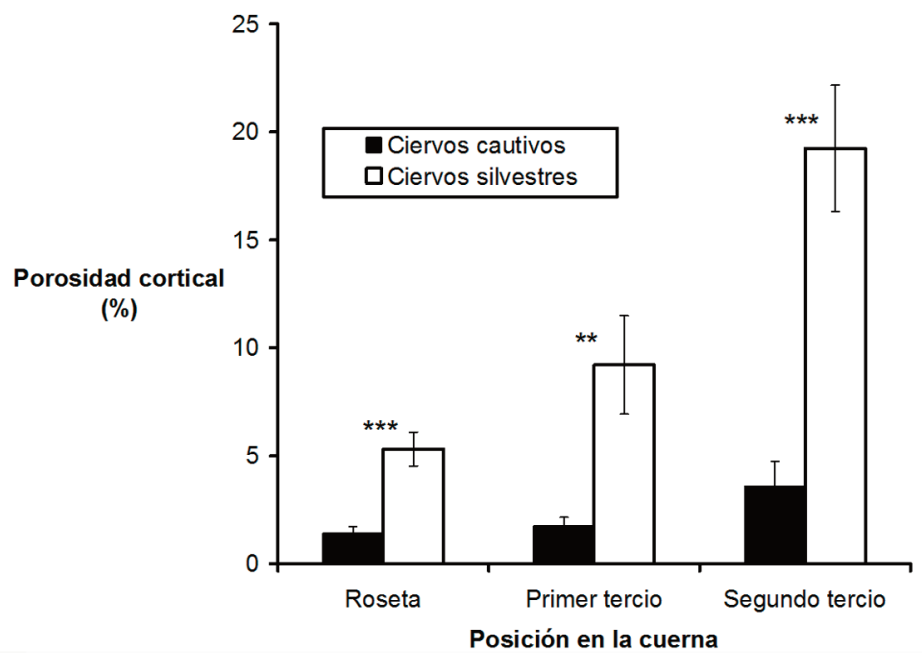

Figura 3. Porosidad del tejido óseo cortical a lo largo de la vara principal de cuernas de ciervo adulto desde la base (roseta) a la punta (de izquierda a derecha). Las barras sólidas corresponden a ciervos de la granja de la UCLM con alimentación de alta calidad (16 \% proteína). Las barras huecas corresponden a ciervos silvestres en un hábitat subóptimo (6-7 \% de proteína).

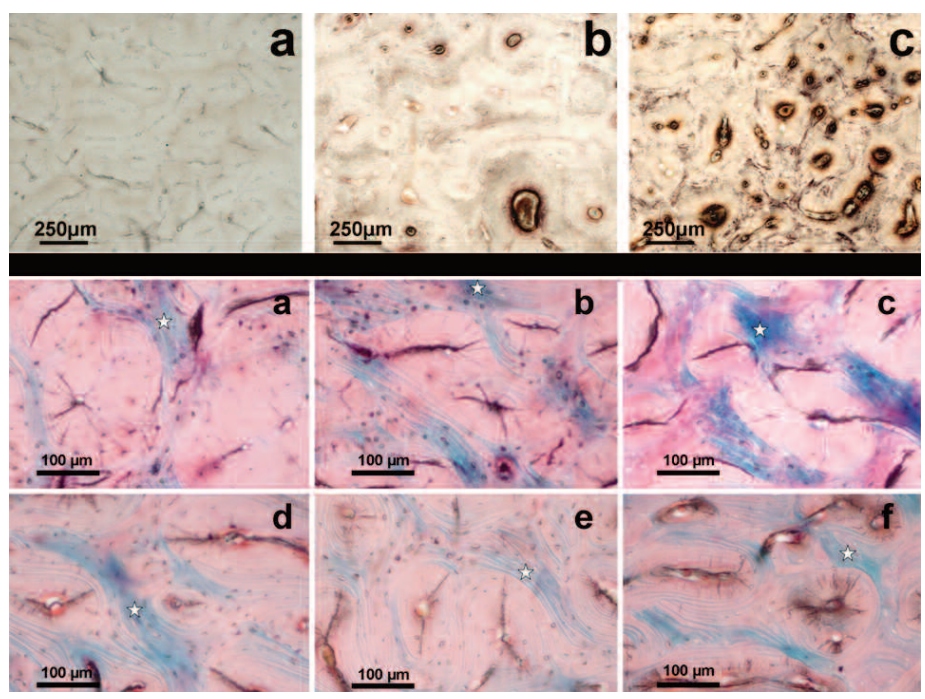

Figura 4. Histoquímica del Zn y K en cuernas de ciervos con distintos tipos de calidad de alimentación. Panel superior, histoquímica mostrando más Zn en zonas más oscuras. El Zn suele estar unido a la fosfatasa alcalina, indicando zonas donde se está mineralizando las osteonas. Las fotografías de osteonas de ciervos con buena alimentación muestran una mineralización completa y pocas zonas con Zn ( $a$, y b superior), mientras que en ciervos silvestres incluso las zonas basales (c superior) que tienen poca porosidad y están más mineralizadas que las partes superiores de la cuerna muestran abundantes zonas con $\mathrm{Zn}$ indicando que están menos mineralizadas que las zonas basales o medias de cuerna de ciervos de granja. Las imágenes de histoquímica del $\mathrm{K}$, inéditas hasta ahora en estudios sobre huesos, mostraron que este mineral (en azul y marcado con asteriscos en algunos sitios) se encuentra en el andamiaje trabecular sobre el que crecen las osteonas (en rosa). Dado que con la maduración del tejido óseo de la cuerna este andamiaje se reduce, las zonas azules son más abundantes en ciervos silvestres ( $d-f$ inferior) que en ciervos de granja con mejor alimentación (a-c inferior).

Para intentar comprender este sorprendente hecho, se llevó a cabo un complejo estudio que se convirtió en el primer paso hacia la comprensión de un problema muy diferente: la osteoporosis. Se seleccionó un coto de caza mayor de unas $1000 \mathrm{Ha}$ en Ciudad Real, donde se habían registrado sistemáticamente durante años las variables climáticas y una gran variedad de medidas de cuernas. A partir de estos registros se encontró que el efecto de rotura apreciado en 2005 era efectivamente una realidad: el $55 \%$ de las cuernas crecidas en 2005 se había roto en más de tres puntas, y 
el $33 \%$ estaban rotas por la vara principal, mientras que las desarrolladas durante el 2004 sólo había un $9 \%$ rotas en la vara principal; además, los pesos de todas las cuernas obtenidas mostraron que las del 2005 eran un $30 \%$ más ligeras que las del año anterior (Landete-Castillejos et al. 2010a). ¿Qué había ocurrido en 2005? El evento excepcional que probablemente explique parte del fenómeno fue el de las bajas temperaturas medias de principios de ese año. Así, las temperaturas mínimas medias de enero y febrero registradas en el coto en donde se realizó el estudio fueron -2.4 y $-2.3^{\circ} \mathrm{C}$, respectivamente; mientras que en 2004 , considerado un año con un invierno de temperaturas dentro de la media (invierno normal, IN), fueron 2.6 y $2.2^{\circ} \mathrm{C}$; en 2005 las temperaturas invernales habían sido excepcionalmente bajas para la zona y la época del año (invierno frío, IF). La granja experimental de ciervos de la Universidad de Castilla-La Mancha está en Albacete, pero a pesar de las bajas temperaturas que también afectaron a la misma, las cuernas no se rompieron ni mostraron los síntomas que se apreciaron en el coto mencionado. Nuestra hipótesis de partida era que esta rotura estaba relacionada con la alimentación, en donde existía una marcada diferencia entre el coto estudiado, con predominio de alimentación natural, y la granja de ciervos, en donde esencialmente el alimento es en base a piensos. Por otra parte, las cuernas empiezan a crecer en marzo (un mes después del periodo de frío), por lo que el efecto tampoco fue causado por la temperatura fría en las cuernas en crecimiento. Esto sugiere que el frío de 2005 podría haber afectado a la vegetación y éstas a la cuerna, con lo que se trataría de un efecto mediado a través de la alimentación herbívora (Estevez et al. 2008), y que habría convertido el hueso más resistente del mundo en un hueso frágil. Las heladas se produjeron a finales de invierno cuando las plantas estaban brotando... ¿qué les sucedió? No hemos podido responder a esta cuestión, porque las cuernas se rompieron durante la berrea del 2005 y los desmogues se recogieron al año siguiente, por lo que no pudimos obtener muestras de plantas a tiempo.

Para evaluar si la nutrición podría haber afectado al rendimiento mecánico de la cuerna, examinamos cuernas rotas en el año 2005 (cuernas IF) y cuernas intactas del invierno anterior, caracterizado por temperaturas dentro de la media (cuernas IN). Apreciamos que algunas cuernas IN se rompieron, mientras que algo menos de la mitad de las cuernas IF estaban intactas. Con el fin de examinar el efecto del frío y no el efecto de ruptura usamos cuernas intactas y rotas de cada año, y examinamos los efectos por separado. Parte de la razón por la que se rompían las cuernas IF parecía ser arquitectónica: las cuernas de ese invierno frío tenían un grosor cortical de hasta $2 \mathrm{~mm}$ inferior al de cuernas del invierno anterior (30\% menos; Tabla 2). La calidad mecánica del material óseo también mostró diferencias: las cuernas IF necesitaban un $27 \%$ menos de energía para romperse que las muestras de cuernas IN (y un $10 \%$ menos de trabajo de fractura).

¿Qué había pasado con la composición de la cuerna? Hubo una pequeña reducción en calcio del año estándar respecto al año frío (de 21 a $20 \%$ ), un cambio aún más pequeño en el fósforo (del $10.1 \%$ al $9.8 \%$ ), y unos pocos cambios en otros minerales (sodio, hierro y cobalto), pero el cambio más marcado tuvo lugar en el silicio. El contenido de silicio en cuernas IF duplicaba al de las cuernas IN. La literatura sobre fisiología vegetal nos revela que las plantas elevan el contenido de silicio en respuesta a algún tipo de estrés: sequía, frío, calor, ataques de insectos, bacterias, o exceso de sodio y manganeso (Ma 2004; Ma y Yamaji 2006; Liang et al. 2007). El efecto más interesante es que una vez toman el silicio, reducen el contenido de sodio o manganeso, aún cuando el efecto desencadenante de la absorción de silicio sea otra causa de estrés y no el exceso de estos minerales.

Lo que encontramos (Landete-Castillejos et al. 2010a) fue que en las plantas del año frío el contenido de silicio de las cuernas era el doble que el del año estándar. Como resultado, las plantas redujeron la absorción de manganeso y el menor contenido en manganeso de la dieta se reflejó en la composición de las cuernas. Sin embargo, el efecto más interesante no es que las cuernas del año frío tuvieran menos manganeso, sino que las cuernas rotas de ambos años tenían mucho menos manganeso que las cuernas intactas. En resumen, si el silicio reflejaba el estrés por frío en las plantas, el menor contenido en manganeso estaba asociado a cuernas rotas de cualquier año. Esto parece estar corroborado por el hecho de que los ciervos bien alimentados de la granja de la universidad tenían cuernas grandes, pero cuyo material era más fácil de romper que el de cuernas de otra finca con deficiencias en minerales pero con un mayor contenido en manganeso. Consecuentemente podemos afirmar que el manganeso refuerza el material óseo. Este fue un hallazgo clave para elaborar una teoría alternativa sobre el origen de la osteoporosis (Landete-Castillejos et al. 2012b), pero también tiene implicaciones en la gestión cinegética: la deficiencia de manganeso puede reducir un $30 \%$ el peso de las cuernas, luego su suplementación podría mejorarlas en casi un tercio. Para muchos gestores, eso supondría un aumento enorme en la rentabilidad de su coto.

\section{El efecto de la gestión pública y privada en las cuernas}

La gestión cinegética en fincas privadas suele diferir de la realizada en territorios cinegéticos públicos, estén o no protegidos con alguna figura. En la gestión cinegética privada suele tomarse como primera medida de gestión el vallado del coto a gestionar (LandeteCastillejos et al. 2010b). Hay distintas razones para ello: el vallado permite implementar un plan propio de gestión (sea para obtener muchos trofeos de calidad media, o un menor número, pero de gran calidad), reduce los daños a cultivos cercanos, impide perder animales de gran valor que podrían ser abatidos en otros terrenos, dificulta (pero no impide) el furtivismo, etc. A la hora de gestionar cualquier población animal cuando ya prácticamente solo queda el hombre como único depredador, una de las medidas fundamentales es el control de la población. Esta es la parte de gestión común en cotos privados o reservas públicas (salvo que sean tan grandes y con suficientes depredadores como para no necesitar capturaderos o caza selectiva). La diferencia entre muchos cotos privados y públicos es que, normalmente, en los primeros se ofrece a los animales alimentación suplementaria, correctores minerales, y a menudo, puntos de agua. España es uno de los principales mercados europeos para las empresas de construcción e instalación de vallados, si bien estos, junto a la suplementación alimenticia y la creación de puntos de agua, son comunes en la gestión cinegética de muchos otros países, tanto de América Latina como Europa (Landete-Castillejos, datos sin publicar).

¿Tiene realmente razón de ser el aportar alimento a los ciervos si el medio se supone que les ofrece todo lo que precisan? ¿Se suplementa para mejorar la condición corporal de los animales a unas densidades excesivas? ¿O los ciervos de cotos con alimentación suplementaria equilibran deficiencias minerales que no pueden compensarse reduciendo la densidad poblacional?

Para contestar a estas preguntas realizamos un estudio comparando una serie de cuernas de 4 cotos privados, 3 reservas públicas (incluyendo un parque nacional), y cuernas de referencia de dos granjas (la de la UCLM y otra privada) donde la alimentación es de alta calidad y equilibrada en casi todos los minerales (Landete-Castillejos et al. 2013).

Los resultados mostraron una fuerte influencia del tipo de gestión pública o privada en la composición de la cuerna, propiedades mecánicas del material óseo, y variables estructurales. La Tabla 3 muestra las variables de la cuerna en reservas públicas, cotos privados y granjas de referencia. Hay diferencias significativas en casi todas las variables examinadas. En la mayor parte de los casos, las cuernas de cotos privados no diferían de los valores de referencia (granjas). Sin embargo las cuernas de reservas bajo gestión pública tenían menores valores de longitud, espesor cortical, densidad o gravedad específica, resistencia arquitectónica a la flexión (Imax, Imin), trabajo de fractura, cenizas y los minerales $\mathrm{Ca}, \mathrm{Mg}$, $\mathrm{Na}, \mathrm{S}$ y $\mathrm{Zn}$. Una de las reservas publicas tenía una densidad de animales muy baja (15 ciervos/100 ha), lo que permitía una com- 
Tabla 2. Composición media ( \pm SE), propiedades mecánicas y estructura de cuernas y material óseo crecido en un mismo coto tras un año de invierno normal (IN) o uno con un periodo de heladas excepcionales al final (IF) que produjo una alta incidencia de roturas. La tabla muestra los datos de todas las cuernas recogidas (99 en IF y 89 en IN), el peso corporal de ciervos abatidos durante estos años (14 en IF y 17 en IN), y las características de una submuestras de cuernas (13 rotas y 7 intactas en IF frente a 15 intactas y 5 rotas en IN).

\section{IN - $2004 \quad$ IF - $2005 \quad$ P}

Todas las cuernas de desmogue

\begin{tabular}{|c|c|c|c|}
\hline Fracturas en la vara principal & 8 rotas & & \\
\hline 81 intactas & 33 rotas & & \\
\hline 66 intactas & $<0.001$ & & \\
\hline $\begin{array}{l}\text { FD: Fuertes daños ( } \geq 3 \text { puntas } \\
\text { rotas o la vara principal) }\end{array}$ & $\begin{array}{l}22 \mathrm{FD} \\
67 \text { no FD }\end{array}$ & $\begin{array}{c}55 \mathrm{FD} \\
44 \text { no FD }\end{array}$ & $<0.001$ \\
\hline Peso de la cuerna, $\mathrm{g}$ & $1449 \pm 66$ & $1106 \pm 44$ & $<0.001$ \\
\hline \multicolumn{4}{|c|}{ Ciervos abatidos } \\
\hline Peso total, kg & $153 \pm 19$ & $165 \pm 5$ & 0.074 \\
\hline Peso en canal, kg & $103 \pm 18$ & $113 \pm 13$ & 0.096 \\
\hline $\begin{array}{l}\text { Cuernas rotas ( } \geq 3 \text { puntas) } \\
\text { frente a intactas }\end{array}$ & $36 \%$ & $55 \%$ & $>0.05$ \\
\hline
\end{tabular}

Cuernas rotas e intactas selecionadas para estudio detallado

\begin{tabular}{lccc}
\hline Espesor cortical, mm & $5.65 \pm 0.28$ & $4.65 \pm 0.2$ & 0.005 \\
Densidad física, g/cm ${ }^{3}$ & $1.750 \pm 0.006$ & $1.722 \pm 0.010$ & 0.027 \\
Diámetro medio, mm & $4.14 \pm 0.11$ & $3.85 \pm 0.06$ & 0.023 \\
Energía de impacto, kJ/m² & $54.9 \pm 2.7$ & $40.1 \pm 1.8$ & 0.001 \\
Modulo de Young's E, GPa & $15.69 \pm 0.32$ & $15.22 \pm 0.39$ & $>0.1$ \\
Fuerza de rotura, MPa & $306.6 \pm 6.4$ & $299.0 \pm 7.6$ & $>0.1$ \\
Trabajo de fractura, kJ/m² & $38.0 \pm 1.5$ & $34.2 \pm 1.1$ & 0.045 \\
Ceniza, \% & $62.3 \pm 0.2$ & $61.5 \pm 0.5$ & 0.095 \\
Ca, \% & $21.0 \pm 0.2$ & $20.3 \pm 0.2$ & 0.012 \\
$\mathrm{P}, \%$ & $10.1 \pm 0.1$ & $9.8 \pm 0.1$ & 0.033 \\
$\mathrm{Mg}, \%$ & $0.464 \pm 0.007$ & $0.462 \pm 0.005$ & $>0.1$ \\
$\mathrm{Na}, \%$ & $0.591 \pm 0.007$ & $0.567 \pm 0.004$ & 0.004 \\
$\mathrm{~K}, \mathrm{mg} / \mathrm{kg}$ & $578 \pm 32$ & $549 \pm 17$ & $>0.1$ \\
$\mathrm{Sr}, \mathrm{mg} / \mathrm{kg}$ & $213 \pm 8$ & $212 \pm 6$ & $>0.1$ \\
$\mathrm{Si}, \mathrm{mg} / \mathrm{kg}$ & $54 \pm 4$ & $100 \pm 11$ & 0.001 \\
$\mathrm{Mn}, \mathrm{mg} / \mathrm{kg}$ & $4.48 \pm 0.25$ & $3.52 \pm 0.42$ & 0.056 \\
$\mathrm{Cu}, \mathrm{mg} / \mathrm{kg}$ & $0.258 \pm 0.014$ & $0.290 \pm 0.012$ & 0.093 \\
$\mathrm{Fe}, \mathrm{mg} / \mathrm{kg}$ & $23.1 \pm 1.2$ & $29.3 \pm 2.2$ & 0.019 \\
$\mathrm{Zn}, \mathrm{mg} / \mathrm{kg}$ & $57.9 \pm 1.7$ & $55.8 \pm 1.6$ & $>0.1$ \\
$\mathrm{~B}, \mathrm{mg} / \mathrm{kg}$ & $2.94 \pm 0.21$ & $2.68 \pm 0.09$ & $>0.1$ \\
$\mathrm{Co}, \mathrm{mg} / \mathrm{kg}$ & $0.224 \pm 0.014$ & $0.169 \pm 0.018$ & 0.024 \\
\hline
\end{tabular}

paración con los cotos privados para ver si la baja densidad compensaba las deficiencias minerales de otras reservas públicas donde los animales viven a densidades de población mayores. La Tabla 4 muestra esta comparación. Los resultados mostraron que una baja densidad no impidió que las cuernas de este parque natural tuvieran menores valores de densidad, trabajo de fractura, $\mathrm{Ca}$, $\mathrm{Mg}, \mathrm{Na}, \mathrm{Cu}, \mathrm{K}, \mathrm{S}$ y $\mathrm{Zn}$, aunque sí tuvo mayores valores de rigidez, fuerza de rotura, Fe, Mn y Si.
Tabla 3. MANOVA indicando diferencias debidas a la gestión en características estructurales, propiedades mecánicas y composición mineral de cuernas de ciervos mantenidos en reservas públicas o cotos privados (con suplementación). Se incluyen como referencia cuernas de la UCLM y de una granja privada donde los ciervos son alimentados casi exclusivamente con pienso. $\mathrm{Ns}=$ no significativo.

\begin{tabular}{|c|c|c|c|c|}
\hline $\begin{array}{c}\text { Características } \\
\text { de la cuernas }\end{array}$ & Publica* & Privada & Referencia & $\mathbf{P}$ \\
\hline \multicolumn{5}{|c|}{ Características estructurales (Wilk's Lambda $=0.160, p<0.001$ ) } \\
\hline $\begin{array}{l}\text { Longitud cuerna } \\
\qquad(\mathrm{cm})\end{array}$ & $66.0 \pm 2.5^{\mathrm{a}}$ & $77.3 \pm 2.0^{\mathrm{b}}$ & $78.6 \pm 1.5^{b}$ & 0.001 \\
\hline EC**: Radio total & $0.40 \pm 0.01^{\mathrm{a}}$ & $0.45 \pm 0.01^{b}$ & $0.50 \pm 0.01^{\mathrm{c}}$ & $<0.001$ \\
\hline $\mathrm{EC}(\mathrm{mm})$ & $3.60 \pm 0.17^{a}$ & $4.66 \pm 0.18^{b}$ & $5.49 \pm 0.14^{c}$ & $<0.001$ \\
\hline $\begin{array}{c}\text { Gravedad } \\
\text { específica }\left(\mathrm{g} \mathrm{cm}^{-3}\right)\end{array}$ & $0.89 \pm 0.02^{\mathrm{a}}$ & $0.99 \pm 0.02^{b}$ & $1.32 \pm 0.02^{c}$ & $<0.001$ \\
\hline \multicolumn{5}{|c|}{ Propiedades mecánicas (Wilk's Lambda $=0.367, p<0.001$ ) } \\
\hline $\operatorname{lmin}$ & $3.78 \pm 0.38^{a}$ & $6.12 \pm 0.52^{b}$ & $5.67 \pm 0.52^{b}$ & $<0.001$ \\
\hline $\operatorname{lmax}$ & $5.45 \pm 0.55^{\mathrm{a}}$ & $8.74 \pm 0.72^{b}$ & $9.19 \pm 0.97^{b}$ & $<0.001$ \\
\hline $\mathrm{E}(\mathrm{GPa})$ & $15.6 \pm 0.2^{\mathrm{a}}$ & $14.2 \pm 0.2^{\mathrm{b}}$ & $14.8 \pm 0.2^{\mathrm{a}, \mathrm{b}}$ & 0.006 \\
\hline $\mathrm{FR}(\mathrm{MPa})$ & $299 \pm 4$ & $282 \pm 5$ & $286 \pm 4$ & Ns \\
\hline $\mathrm{TF}\left(\mathrm{kJ} \mathrm{m} \mathrm{m}^{-2}\right)$ & $35.3 \pm 0.8^{a}$ & $44.2 \pm 0.8^{b}$ & $42.3 \pm 0.6^{b}$ & $<0.001$ \\
\hline
\end{tabular}

Composición química (Wilk's Lambda $=0.008, \mathrm{p}<0.001$ )

\begin{tabular}{ccccc} 
ceniza (\%) & $59.8 \pm 0.2^{\mathrm{a}}$ & $60.8 \pm 0.2^{\mathrm{a}, \mathrm{b}}$ & $61.4 \pm 0.3^{\mathrm{b}}$ & 0.004 \\
$\mathrm{Ca}(\%)$ & $19.9 \pm 0.1^{\mathrm{a}}$ & $20.6 \pm 0.1^{\mathrm{b}}$ & $20.5 \pm 0.1^{\mathrm{a}, \mathrm{b}}$ & 0.013 \\
$\mathrm{Mg}(\%)$ & $0.41 \pm 0.00^{\mathrm{a}}$ & $0.46 \pm 0.01^{\mathrm{b}}$ & $0.51 \pm 0.01^{\mathrm{c}}$ & $<0.001$ \\
$\mathrm{Na}(\%)$ & $0.47 \pm 0.00^{\mathrm{a}}$ & $0.65 \pm 0.02^{\mathrm{b}}$ & $0.55 \pm 0.01^{\mathrm{c}}$ & $<0.001$ \\
$\mathrm{P}(\%)$ & $9.37 \pm 0.06^{\mathrm{a}}$ & $9.58 \pm 0.07^{\mathrm{a}}$ & $9.96 \pm 0.06^{\mathrm{b}}$ & $<0.001$ \\
$\mathrm{~B}(\mathrm{ppm})$ & $2.86 \pm 0.06$ & $2.87 \pm 0.08$ & $3.31 \pm 0.31$ & $\mathrm{Ns}$ \\
$\mathrm{Co}(\mathrm{ppm})$ & $0.04 \pm 0.01^{\mathrm{a}}$ & $0.03 \pm 0.01^{\mathrm{a}}$ & $0.29 \pm 0.03^{\mathrm{b}}$ & $<0.001$ \\
$\mathrm{Cu}(\mathrm{ppm})$ & $0.36 \pm 0.03$ & $0.48 \pm 0.05$ & $0.42 \pm 0.06$ & $\mathrm{Ns}$ \\
$\mathrm{Fe}(\mathrm{ppm})$ & $47.7 \pm 4.6^{\mathrm{a}}$ & $14.8 \pm 2.8^{\mathrm{b}}$ & $24.8 \pm 2.7^{\mathrm{b}}$ & $<0.001$ \\
$\mathrm{~K}(\mathrm{ppm})$ & $523 \pm 24$ & $475 \pm 16$ & $454 \pm 21$ & $\mathrm{Ns}$ \\
$\mathrm{Mn}(\mathrm{ppm})$ & $12.2 \pm 0.7^{\mathrm{a}}$ & $3.02 \pm 0.67^{\mathrm{b}}$ & $1.40 \pm 0.12^{\mathrm{c}}$ & $<0.001$ \\
$\mathrm{~S}(\mathrm{ppm})$ & $917 \pm 24^{\mathrm{a}}$ & $1340 \pm 62^{\mathrm{b}}$ & $912 \pm 30^{\mathrm{a}}$ & $<0.001$ \\
$\mathrm{Se}(\mathrm{ppm})$ & $0.71 \pm 0.05^{\mathrm{a}}$ & $0.77 \pm 0.09^{\mathrm{a}}$ & $1.43 \pm 0.28^{\mathrm{b}}$ & $\mathrm{Ns}$ \\
$\mathrm{Si}(\mathrm{ppm})$ & $182 \pm 19^{\mathrm{a}}$ & $40.5 \pm 6.8^{\mathrm{b}}$ & $118 \pm 11^{\mathrm{a}}$ & $<0.001$ \\
$\mathrm{Sr}(\mathrm{ppm})$ & $159 \pm 5^{\mathrm{a}}$ & $195 \pm 6^{\mathrm{a}, \mathrm{b}}$ & $360 \pm 50^{\mathrm{b}}$ & 0.003 \\
$\mathrm{Zn}(\mathrm{ppm})$ & $45.2 \pm 1.4^{\mathrm{a}}$ & $61.4 \pm 1.5^{\mathrm{b}}$ & $60.1 \pm 3.2^{\mathrm{b}}$ & $<0.001$ \\
\hline & & & &
\end{tabular}

* Diferentes superíndices $\left({ }^{a},{ }^{b} y^{c}\right)$ indican diferencias estadísticas entre grupos. Así, por ejemplo, la longitud de la cuerna es diferente en una reserva pública comparada con la privada y de referencia, que no difieren entre sí.

**EC significa espesor cortical; I es una propiedad que mide la resistencia arquitectónica a la flexión, el segundo momento de área (en su diámetro mayor o menor); E es el módulo de Young de elasticidad; FR es fuerza de rotura; TF es trabajo de fractura.

\section{Efectos en huesos internos y sus aplicaciones a la conservación}

Hemos visto una serie de estudios sobre la composición de la cuerna que pueden tener claras implicaciones en gestión cinegética, e incluso, indirectamente, en aspectos médicos; pero también pueden tener relevancia en conservación de especies amenazadas. Realizamos un estudio sobre fémures de ciervas mantenidas 
Tabla 4. MANOVA indicando diferencias en características estructurales, propiedades mecánicas y composición mineral de cuernas de ciervos procedentes de un parque nacional (CB) en donde se encuentran en bajas densidades (unos 15 ciervos/100 Ha) y ciervos mantenidos en cotos vallados de caza privados en donde se utilizan complementos alimentarios y suplementos minerales. $\mathrm{Ns}=$ no significativo.

\begin{tabular}{|c|c|c|c|}
\hline $\begin{array}{l}\text { Características } \\
\text { de la cuernas }\end{array}$ & CB & Privada & $P$ \\
\hline \multicolumn{4}{|c|}{ Características estructurales (Wilk's Lambda $=0.804, p<0.090$ ) } \\
\hline Longitud cuerna $(\mathrm{cm})$ & $84.0 \pm 2.2$ & $77.3 \pm 2.0$ & Ns \\
\hline EC:Radio total & $0.42 \pm 0.01$ & $0.45 \pm 0.01$ & Ns \\
\hline$E C(\mathrm{~mm})$ & $4.70 \pm 0.20$ & $4.66 \pm 0.18$ & Ns \\
\hline $\begin{array}{c}\text { Gravedad } \\
\text { específica }\left(\mathrm{g} \mathrm{cm}^{-3}\right)\end{array}$ & $0.92 \pm 0.02$ & $0.99 \pm 0.02$ & $<0.001$ \\
\hline \multicolumn{4}{|c|}{ Propiedades mecánicas (Wilk's Lambda $=0.477, p=0.001$ ) } \\
\hline$I_{\min }$ & $6.61 \pm 0.54$ & $6.12 \pm 0.52$ & Ns \\
\hline$I_{\max }$ & $9.49 \pm 0.76$ & $8.74 \pm 0.72$ & Ns \\
\hline$E(\mathrm{GPa})$ & $15.7 \pm 0.3$ & $14.2 \pm 0.2$ & 0.034 \\
\hline$F R(\mathrm{MPa})$ & $310 \pm 3$ & $282 \pm 5$ & Ns \\
\hline$T F\left(\mathrm{~kJ} \mathrm{~m}^{-2}\right)$ & $36.5 \pm 1.1$ & $44.2 \pm 0.8$ & $<0.001$ \\
\hline
\end{tabular}

Composición química (Wilk's Lambda $=0.034, \mathrm{p}<0.001$ )

$\begin{array}{cccc}\text { ceniza (\%) } & 60.0 \pm 0.3 & 60.8 \pm 0.2 & \text { Ns } \\ \mathrm{Ca}(\%) & 20.1 \pm 0.2 & 20.6 \pm 0.1 & \mathrm{Ns} \\ \mathrm{Mg}(\%) & 0.40 \pm 0.01 & 0.46 \pm 0.01 & <0.001 \\ \mathrm{Na}(\%) & 0.46 \pm 0.01 & 0.65 \pm 0.02 & <0.001 \\ \mathrm{P}(\%) & 9.46 \pm 0.09 & 9.58 \pm 0.07 & \mathrm{Ns} \\ \mathrm{B}(\mathrm{ppm}) & 2.90 \pm 0.10 & 2.87 \pm 0.08 & \mathrm{Ns} \\ \mathrm{Co}(\mathrm{ppm}) & 0.03 \pm 0.01 & 0.03 \pm 0.01 & \mathrm{Ns} \\ \mathrm{Cu}(\mathrm{ppm}) & 0.27 \pm 0.02 & 0.48 \pm 0.05 & \mathrm{Ns} \\ \mathrm{Fe}(\mathrm{ppm}) & 31.1 \pm 3.4 & 14.8 \pm 2.8 & \mathrm{Ns} \\ \mathrm{K}(\mathrm{ppm}) & 407 \pm 7 & 475 \pm 16 & 0.030 \\ \mathrm{Mn}(\mathrm{ppm}) & 11.7 \pm 0.9 & 3.02 \pm 0.67 & <0.001 \\ \mathrm{~S}(\mathrm{ppm}) & 840 \pm 10 & 1340 \pm 62 & 0.001 \\ \mathrm{Se}(\mathrm{ppm}) & 0.74 \pm 0.06 & 0.77 \pm 0.09 & \mathrm{Ns} \\ \mathrm{Si}(\mathrm{ppm}) & 115 \pm 10 & 40.5 \pm 6.8 & 0.001 \\ \mathrm{Sr}(\mathrm{ppm}) & 179 \pm 7 & 195 \pm 6 & 0.010 \\ \mathrm{Zn}(\mathrm{ppm}) & 52.6 \pm 2.0 & 61.4 \pm 1.5 & 0.012\end{array}$

durante 3 años en un cercado de 13 Ha (con 13 ciervas) sin más comida que la de las plantas, para compararlas con otro cercado igual en el que las ciervas fueron suplementadas con un alimento altamente nutritivo (22 \% de proteína y suplementación de casi todos los minerales esenciales). Los resultados (Olguin et al., 2013) indican que la composición de los huesos refleja la composición de microminerales en la dieta ( $\mathrm{Mn}, \mathrm{Cu}$ y $\mathrm{Zn}$, pero también otros), pero no la de macrominerales (como $\mathrm{Na}$ o $\mathrm{P}$ ).

¿Podrían usarse estos hallazgos para la conservación y gestión de ecosistemas, incluso en donde no haya necesariamente cérvidos? La composición de los huesos ya ha sido ampliamente estudiada para detectar la existencia de minerales contaminantes que se almacenan en tejidos óseos (v.g., en cuernas, Kierdorf y Kierdorf
2006). Pero el estudio de los huesos podría permitir saber si el exceso de algunos minerales podría producir la deficiencia de otros, y que estos estuviesen a su vez haciendo peligrar aún más la supervivencia de poblaciones de especies amenazadas; así, por ejemplo, el exceso de azufre y molibdeno reducen la absorción de cobre por la formación de tiomolibdatos de cobre que impiden la asimilación de este último mineral, induciendo una deficiencia incluso si los niveles de cobre en el medio son adecuados; McDowell 2003). Existe evidencia reciente de que los ciervos evitan ciertas plantas con alto contenido en azufre, aunque posean también otros minerales necesarios ( $T$, Landete-Castillejos, datos sin publicar).

En suma, podemos afirmar que los estudios sobre composición, propiedades mecánicas, estructura e histología de cuernas en relación con variables ecológicas, e incluso sanitarias, abren todo un campo que podría ser útil en muchas otras especies animales.

\section{Referencias}

Baxter, B.J., Andrews, R.N., Barrell, G.K. 1999. Bone turnover associated with antler growth in red deer (Cervus elaphus). Anatomical Record 256:14-19

Clutton-Brock, T.H., Albon, S.D., Gibson, R.M., Guinness, F.E. 1979. The logical stag: adaptive aspects of fighting in red deer (Cervus elaphus L.). Animal Behaviour 27:211-225.

Clutton-Brock, T.H., Guinness, F.E., Albon, S.D. 1982. Red deer. Behaviour and ecology of two sexes. Edinburgh University Press, Edinburgh, Reino Unido.

Clutton-Brock, T.H., Albon, S.D., Guinness, F.E. 1988. Reproductive success in male and female red deer. En: Clutton-Brock, T.H. (ed). Reproductive Success, pp. 325-343. University of Chicago Press, Chicago, USA.

Currey, J.D. 2002. Bones: Structure and Mechanics. Princeton University Press, Princeton, EE.UU.

Currey, J.D., Landete-Castillejos, T., Estevez, J., Ceacero, F., Olguin, A., Garcia, A., Gallego, L. 2009. The mechanical properties of deer antler bone when used in fighting. Journal of Experimental Biology 212:398593.

Davison, S.K., Siminoski, K., Adachi, J.D., Hanley, D.A., Goltzman, D., Hodsman, A.B., Josse, R., Kaiser, S., Olskynski, P., Papaioannou, A., Ste-Marie, L.G., Kendler, D.L., Tenenhouse, A., Brown, J.P. 2006. Bone strength: The whole is greater than the sum of its parts. Semin Arthritis Rheu 36:22-31.

Estévez, J.A., Landete-Castillejos, García, A.J., Ceacero, F., Gallego, L. 2008. Maintenance regime and bone structural effects in composition and radioopacity of Iberian red deer antlers (Cervus elaphus hispanicus). European Journal of Wildlife Research 54:215-223

Estévez, J.A., Landete-Castillejos, T., Martínez, A., García, A.J., Ceacero, F., Gaspar-López, E., Calatayud, A., Gallego, L. 2009. Antler mineral composition of Iberian red deer Cervus elaphus hispanicus is related to mineral profile of diet. Acta Theriologica 54:235-242

Gaspar-Lopez, E., Landete-Castillejos, T., Estevez, J.A., Ceacero, F., Gallego, L., Garcia, A.J. 2010. Biometrics, testosterone, cortisol and antler growth cycle in Iberian red deer stags (Cervus elaphus hispanicus). Reproduction in Domestic Animals 45:243-249

Gebert, C., Verheyden-Tixier, H. 2001. Variations of diet composition of red deer (Cervus elaphus L.) in Europe. Mammal Review 31:189-201.

Gomez, J.A., Ceacero, F., Landete-Castillejos, T., Gaspar-López, E., García, A.J., Gallego, L. 2012. Factors affecting antler investment in yearlings, subadults and adults of Iberian deer. Animal Production Science 52:867873.

Gomez, S., Garcia, A.J., Luna, S., Kierdorf, U., Kierdorf, H., Gallego, L., Landete-Castillejos, T. 2013. Labeling studies on cortical bone formation in the antlers of red deer (Cervus elaphus). Bone 52:506-515.

Goss, R.J. 1983. Deer antlers: regeneration, function, and evolution. Academic Press. New York, USA

Huxley, J. 1931. The relative size of antlers of deer. Proceedings of the Zoological Society of London :819-864.

Kierdorf, U., Kierdorf, H. 2006. Roe and red deer antlers as bioindicators of pollution of deer habitats by lead and fluoride. Veterinarski Arhiv 76:S117-S129.

Landete-Castillejos, T., Garcia, A.J., Gallego, L. 2007a. Body weight, early growth and antler size influence antler bone mineral composition of Iberian Red Deer (Cervus elaphus hispanicus). Bone 40:230-235. 
Landete-Castillejos, T., Estevez, J.A., Martínez, A., Ceacero, F., Garcia, A.J., Gallego, L. 2007b. Does chemical composition of antler bone reflect the physiological effort made to grow it? Bone 40:1095-1102.

Landete-Castillejos, T., Currey, J.D., Estevez, J.A., Gaspar-López, E., García, A.J., Gallego, L. 2007c. Influence of physiological effort of growth and chemical composition on antler bone mechanical properties. Bone 41:794-803.

Landete-Castillejos, T., Estevez, J.A., Fierro, Y., Calatayud, A., Ceacero, F., Garcia, A.J., Gallego, L. 2010a. Do drastic weather effects on diet influence changes in chemical composition, mechanical properties and structure in deer antlers? Bone 47:815-825.

Landete-Castillejos, T., Gallego, L., Estévez, J.A., Garcia, A.J., Fierro, Y. $2010 \mathrm{~b}$. Fencing of game estates in Spain considered as management unit. En: Chapman, H.G., Hecker, K. (eds) Enclosures: A Dead-End? Influence on Game Biology, Conservation and Hunting, pp. 68-79. International Council for Game and Wildlife Conservation - CIC, Sopron, Hungría.

Landete-Castillejos, T., Currey, J.D., Ceacero, F., García, A.J., Gallego, L., Gomez, S. 2012a. Does nutrition affect bone porosity and mineral tissue distribution in deer antlers? The relationship between histology, mechanical properties and mineral composition. Bone 50:245-254.

Landete-Castillejos, T., Molina-Quilez, I., Estevez, F., Ceacero, J.A., Garcia, A.J., Gallego, L. 2012b. Alternative hypothesis for the origin of osteoporosis: The role of Mn. Frontiers in Bioscience E4:1385-1390.
Landete-Castillejos, T., Estévez, J.A., Ceacero, F., Garcia, A.J., Gallego, L. 2013, en prensa. Effects of public vs. private management on deer antler composition, mechanical and structural variables. European Journal of Wildlife Research 00:000-000.

Liang, Y., Sun, W., Zhu, Y.G., Christie, P. 2007. Mechanisms of silicon-mediated alleviation of abiotic stresses in higher plants: a review. Environmental Pollution 147:422-428.

Ma, J.F. 2004. Role of silicon in enhancing the resistance of plants to biotic and abiotic stresses. Soil Science and Plant Nutrition 50:11-18.

Ma, J.F., Yamaji, N. 2006. Silicon uptake and accumulation in higher plants. Trends in Plant Science 11:392-397.

McDowell, L.R. 2003. Minerals in animal and human nutrition. Elsevier, Amsterdam, Holanda.

Muir, P.D., Sykes, A.R., Barrell, G.K. 1987. Calcium metabolism in red deer (Cervus elaphus) offered herbages during antlerogenesis: kinetic and stable balance studies. Journal of agricultural Science 109: 357-364.

Olguin, C.A., Landete-Castillejos, T., Ceacero, F., Garcia, A.J., Gallego L. 2013, en prensa. Effects of feed supplementation on mineral composition, mechanical properties and structure in femora of Iberian red deer hinds (Cervus elaphus hispanicus). PLoS One 00:000-000.

Topinski, P. 1974. The role of antlers in establishment of the red deer herd hierarchy. Acta Theriologica 19:509-514. 